OPEN ACCESS

Edited by:

Man Li,

Huazhong University of Science and Technology, China

Reviewed by:

Natalia Egorova Brumley,

University of Melbourne, Australia

Rong Zhang,

Peking University, China

*Correspondence:

Xueyong Shen

snowysh@hotmail.com

Sheng Liu

liusheng@shutcm.edu.cn

tThese authors have contributed equally to this work

Specialty section:

This article was submitted to

Perception Science,

a section of the journal

Frontiers in Neuroscience

Received: 30 September 2021 Accepted: 08 November 2021 Published: 07 December 2021

Citation:

Xiang A, Chen M, Qin C, Rong J, Wang C, Shen $X$ and Liu S (2021) Frequency-Specific Blood Oxygen

Level Dependent Oscillations Associated With Pain Relief From Ankle Acupuncture in Patients With

Chronic Low Back Pain

Front. Neurosci. 15:786490. doi: 10.3389/fnins.2021.786490

\section{Frequency-Specific Blood Oxygen Level Dependent Oscillations Associated With Pain Relief From Ankle Acupuncture in Patients With Chronic Low Back Pain}

\author{
Anfeng Xiang ${ }^{1,2 t}$, Meiyu Chen ${ }^{1 \dagger}$, Chuan Qin ${ }^{1 t}$, Jun Rong ${ }^{3}$, Can Wang ${ }^{1}$, Xueyong Shen ${ }^{1 *}$ \\ and Sheng Liu ${ }^{1 *}$ \\ 'School of Acupuncture-Moxibustion and Tuina, Shanghai University of Traditional Chinese Medicine, Shanghai, China, \\ ${ }^{2}$ The First Rehabilitation Hospital of Shanghai, School of Medicine, Tongji University, Shanghai, China, ${ }^{3}$ Department \\ of Sports Rehabilitation, Zhejiang Integrated Traditional and Western Medicine Hospital, Zhejiang, China
}

Objective: Recent advances in brain imaging have deepened our knowledge of the neural activity in distinct brain areas associated with acupuncture analgesia. However, there has not been conclusive research into the frequency-specific restingstate functional changes associated with acupuncture analgesia in patients with chronic pain. Here, we aimed to characterize changes across multiple frequencies of restingstate cortical activity associated with ankle acupuncture stimulation (AAS) in patients with chronic low back pain (CLBP) and healthy controls.

Methods: Twenty seven patients with CLBP and Twenty five age- and gendermatched healthy volunteers were enrolled in the study. Participants received tactile sham acupuncture (TSA) and AAS, respectively. The whole-brain amplitude of low-frequency fluctuation (ALFF) in the range $0.01-0.25 \mathrm{~Hz}$ was assessed for changes associated with each intervention. Further, a visual analog scale (VAS) was used to collect subjective measures of pain intensity in patients. Linear mixed-effect modeling (LME) was used to examine the mean ALFF values of AAS and TSA between patients and healthy controls.

Results: The ALFF was modulated in the default mode network (an increase in the medial prefrontal cortex, and a decrease in the cerebellum/posterior ingulate/parahippocampus, $P<0.01$, corrected) in both patients and controls. Decreased ALFF in the bilateral insular was frequency-dependent. Modulations in the cerebellum and right insular were significantly correlated with VAS pain score after AAS $(P<0.01)$.

Conclusion: Hence, frequency-specific resting-state activity in the cerebellum and insular was correlated to AAS analgesia. Our frequency-specific analysis of ALFF may provide novel insights related to pain relief from acupuncture.

Keywords: ankle acupuncture stimulation, immediate analgesia, resting-state brain activity, insular, cerebellum 


\section{INTRODUCTION}

Chronic low back pain (CLBP) affects $60-80 \%$ of people at some point in their lifetime and has become one of the most common reasons for visiting a physician (Hoy et al., 2010; Urits et al., 2019). Nearly a third of people who seek treatment for low back pain (LBP) will experience persistent back pain for 1 year after the acute period (Harrisson et al., 2017). Prolonged loss or impairment of function caused by LBP often has an economic impact on the patient, including treatment costs, disability payments, and reduction or loss of productivity. LBP is associated with structural, neurochemical, and functional changes in the brain, in regions such as the thalamus, the anterior insula, and the somatosensory cortex (Gao et al., 2016; Konno and Sekiguchi, 2018). Complex processes induced by peripheral and central sensitization following the experience of pain may be involved in the transition from acute to chronic LBP.

A growing body of evidence supports the use of acupuncture as an effective treatment for acute and chronic pain, including headaches, neck pain, osteoarthritis pain, and LBP (Furlan et al., 2005; Berman et al., 2010; Wang et al., 2021). The efficacy of acupuncture as an analgesic has also been verified using incision pain, and inflammatory and neuropathic pain-related behavioral tests in animal studies (Zhang et al., 2014, 2021). Nevertheless, the neural mechanisms underlying the analgesic effects of acupuncture have not yet been fully investigated. Functional magnetic resonance imaging (fMRI) provides a useful technique to investigate the mechanisms by which processes in the central nervous system are modulated by acupuncture. fMRI studies on acupuncture at commonly used acupoints have demonstrated significant functional response in many brain regions associated with acupuncture analgesia, such as the prefrontal cortex, the limbic system, the paralimbic and subcortical gray structures, and the cerebellum (Hui et al., 2000; Napadow et al., 2005; Wang et al., 2013).

Frequency-specific blood oxygen level dependent (BOLD) signal oscillations measured using resting-state fMRI (rs-fMRI) are of increasing interest, and the nature of this frequencyspecificity has many biological interpretations. Studies reporting specific frequencies of BOLD signal oscillations indicated that each different frequency band contributes uniquely to brain network integration in terms of physiological and pathological activities (Hipp and Siegel, 2015; He et al., 2016). Oscillations within the $0.01-0.25 \mathrm{~Hz}$ range can be consistently divided into the intrinsic frequency bands, norm-1 $(0.01-0.1 \mathrm{~Hz})$, norm-2 (0.01-0.08 Hz), slow-5 (0.01-0.027 Hz), slow-4 (0.027-0.073 Hz), slow-3 (0.073-0.198 Hz), and slow-2 (0.198-0.25 Hz) (Lou et al., 2020). These frequency bands have been shown to be highly reproducible and independent of the fMRI sampling rate. It has been proposed that different frequency bands signify different physiological changes (Yan et al., 2020). For example, BOLD signal oscillations lower than $0.02 \mathrm{~Hz}$ were typically observed in the putamen and higher-frequency oscillations $(>0.08 \mathrm{~Hz})$ in limbic areas (Zuo et al., 2010). As such, a frequency-specific approach may provide more information than conventional approaches (relying predominantly on oscillations $<0.1 \mathrm{~Hz}$ ) to help interpret localized BOLD changes associated with acupuncture stimulation in patients with LBP. To our knowledge, no previous study has assessed the frequency-specific restingstate functional changes associated with acupuncture analgesia in LBP patients.

In the present study, we explored the contribution of different frequency bands to changes in resting-state BOLD fMRI associated with the administration of acupuncture in LPB patients. To detect the frequency-specific functional changes that may play an important role in acupuncture analgesia, we assessed BOLD oscillations in frequency bands (0.01$0.1 \mathrm{~Hz}, 0.01-0.08 \mathrm{~Hz}, 0.01-0.027 \mathrm{~Hz}, 0.027-0.073 \mathrm{~Hz}, 0.073-$ $0.198 \mathrm{~Hz}$, and $0.198-0.25 \mathrm{~Hz}$ ) to compare resting-state activity in LBP patients with that of healthy controls. A painless acupuncture technique, ankle acupuncture stimulation (AAS), was used as the intervention method. AAS is a type of subcutaneous acupuncture that was developed in the 1970s (Zhang, 1997). It has been shown that AAS not only has a measurable analgesic effect in numerous conditions associated with pathological pain such as low back pain, cancer, and postoperative recovery (Zeng et al., 2014; Zhu et al., 2014), but also significantly improves the pain threshold of healthy individuals (Marra et al., 2011). AAS requires the insertion of a single needle near the ankle without inducing any localized sensation of the needle. Its stimulation is mild and comprises merely a tactile sensation on the skin. Therefore, AAS may be helpful in investigating patterns of brain activity specifically associated with acupuncture analgesia by excluding or minimizing the possibility of confounding factors such as somatosensation induced by Deqi needle manipulation in traditional acupuncture methods.

\section{MATERIALS AND METHODS}

\section{Participants}

The study protocol was formulated in accordance with the Declaration of Helsinki and approved by the ethical committee of the Center of Cognitive and Brain Disorders of Hangzhou Normal University (20190102). Our present study was registered on www.chictr.org.cn (Identifier: ChiCTR1800020029). Participants gave written informed consent before undergoing screening.

Patients were assessed by an experienced rehabilitation physician according to the following criteria. The inclusion criteria of patients were as follows: (1) right-handed adults of any gender aged 18 to 65 years; (2) a history of low back pain for 6 months or more; (3) an initial self-reported pain intensity evaluated using the visual analog scale (VAS, $0-10$ ) of at least 4 points; (4) able to understand and complete the consent form and clinical assessment questionnaires without assistance. The inclusion criteria of healthy subjects were as follows: (1) righthanded, age- and gender-matched adults; (2) no history of pain or relevant serious disease; (3) able to understand and complete the consent form without assistance.

The exclusion criteria of patients and healthy subjects were as follows: (1) the presence of another cause of pain unrelated to LBP; (2) having taken any medication or undergone physical therapy in the past week; (3) any other serious illnesses or neuropsychiatric diseases; (4) any contraindications for 
undergoing MRI, including the presence of metal implants, cardiac pacemakers, or claustrophobia; (5) any contraindications for undergoing acupuncture, such as pregnancy or a tendency to bleed easily; (6) a history of sleep deprivation or women who were experiencing their menstrual period; (7) having consumed coffee or alcohol in the $10 \mathrm{~h}$ prior to the MRI scan. Participants were free to withdraw at any time. To ensure data quality, any individual who moved their head by more than $1 \mathrm{~mm}$ or $2^{\circ}$ during the scan was excluded from the analyses.

\section{Randomization and Blinding}

The order of AAS and TSA runs was pseudo-randomized across subjects to reduce order effects. In order to maximize washout of acute stimulation effects, these two runs (AAS or TSA) were separated by structural scans which lasted $15 \mathrm{~min}$. In the present study, participants were acupuncture naïve and were informed that there would be "different forms" of acupuncture during fMRI. In addition, the needles used for AAS and TSA were identical. Our preliminary test showed that the sensations of AAS and TSA were similar. The locations of needle stimulation were taped with medical adhesive tape. Participants lay supine in the scanner with their vision of distal body regions blocked by the MRI head coil, preventing them from viewing the intervention occurring at their periphery. Participants could be blinded to the order of AAS and TSA. Blinding success was validated at the end of the study.

\section{Ankle Acupuncture Stimulation Stimulation}

AAS and TSA were performed by the same experienced acupuncturist using disposable silver acupuncture needles $(0.35 \times 40 \mathrm{~mm}$; Zhongyan Taihe Brand, Beijing, China). The left lower 5th AAS zone was selected as the needle insertion point, with the exact location about three-finger widths above the lateral malleolus, near the posterior edge of the fibula and adjacent tendons (Figure 1). The acupuncture method used was based on that described in "Wrist-Ankle Acupuncture" (Zhang, 1997). Briefly, the participant laid in a supine position and exposed the skin of the lateral side of the left leg. After sterilizing the skin and needle, the acupuncturist inserted the needle into the skin at an angle of about $30^{\circ}$. The needle was angled horizontally into the subcutaneous layer and inserted toward the direction of the knee about $35 \mathrm{~mm}$. Participants did not feel any sensation during AAS if this procedure was correctly conducted. The locations of needle stimulation were taped with medical adhesive tape.

During TSA, participants exposed the skin on the lateral side of the left leg, and the stimulus was administered to the same location as in AAS. Instead of inserting a needle into the skin, the needle was gently placed against the skin and held there for $5 \mathrm{~s}$ without penetrating the skin. All other procedures were identical to those used in AAS.

\section{Functional Magnetic Resonance Imaging Scanning Procedure}

The study was conducted in the MRI room of the Center of Cognitive and Brain Disorders, Hangzhou Normal University.

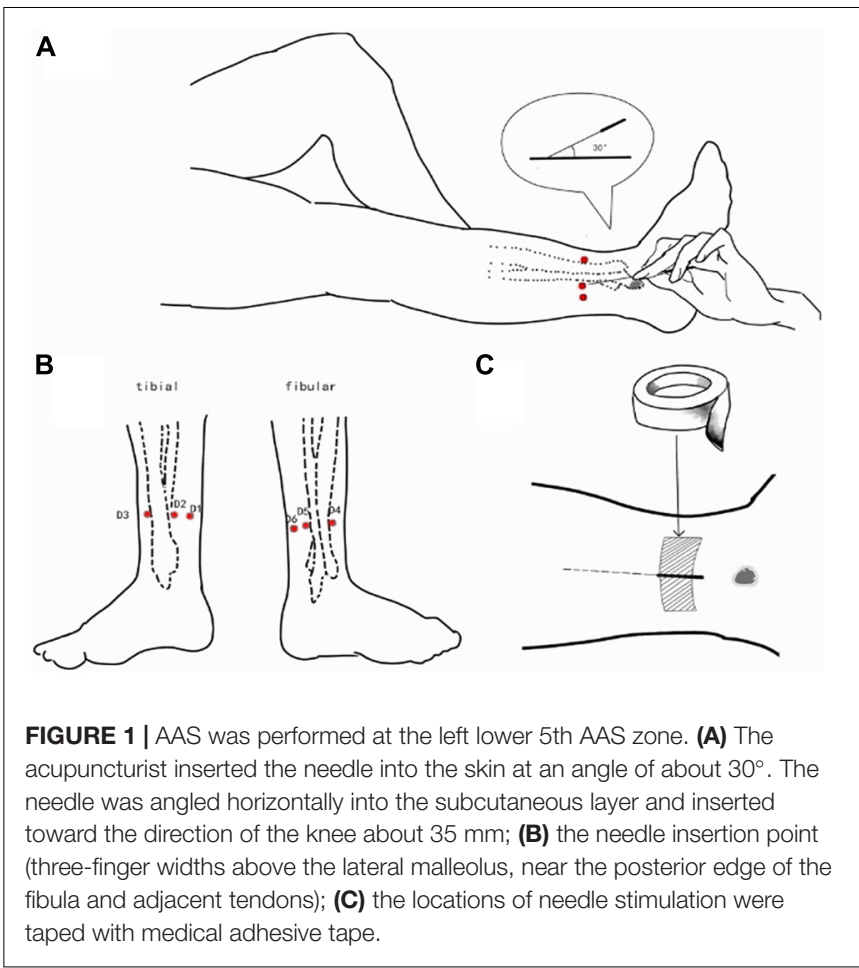

Participants were scanned on a GE-750, 3 T MRI system with an 8-channel head coil. The participants wore earplugs and lay in a supine position on the scanner bed. Sponge padding was used to minimize head movement. During the scanning process, participants were told to keep their eyes closed, stay awake, and try not to think of anything.

The fMRI acquisition used a gradient echo (GRE) echo-planar imaging (EPI) sequence with the following parameters: number of slices $=43$, acquired interleaved, matrix size $=64 \times 64$, repetition time $(\mathrm{TR})=2,000 \mathrm{~ms}$, echo time $(\mathrm{TE})=30 \mathrm{~ms}$, flip angle $(\mathrm{FA})=90^{\circ}$, slice thickness $=3.2 \mathrm{~mm}$, voxel size $=3.4 \times 3.4 \times 3.2 \mathrm{~mm}^{1}$. A total of 240 whole-brain volumes were obtained. A 3D T1-weighted structural image was acquired using a spoiled gradient echo (SPGR) sequence, as follows: number of slices $=176$, matrix size $=256 \times 256$, $\mathrm{TR}=8,100 \mathrm{~ms}, \mathrm{TE}=3.1 \mathrm{~ms}, \mathrm{FA}=8^{\circ}$, slice thickness $=1 \mathrm{~mm}$, voxel size $=1 \times 1 \times 1 \mathrm{~mm}^{3}$, inversion time $=450 \mathrm{~ms}$, and bandwidth $=31.25 \mathrm{~Hz}$. Each participant received one functional image scan following administration of either the TSA or AAS (all participants received both TSA and AAS). The structural image was acquired between the two functional runs.

fMRI scanning paradigm is shown in Figure 2. Similar to the study of Dhond et al. (2008), the order of TSA and AAS runs was pseudo-randomized across subjects. The TSA and AAS portions of the scan session were separated by functional and structure image scan lasting $15 \mathrm{~min} .8 \mathrm{~min}$ rest run was completed before, and after each stimulation run. During rest runs, subjects were asked to lie quietly, keep their eyes closed and stay awake.

\footnotetext{
${ }^{1}$ www.rfmri.org/dpabi
} 


\section{fMRI SCANNING PROCEDURE}

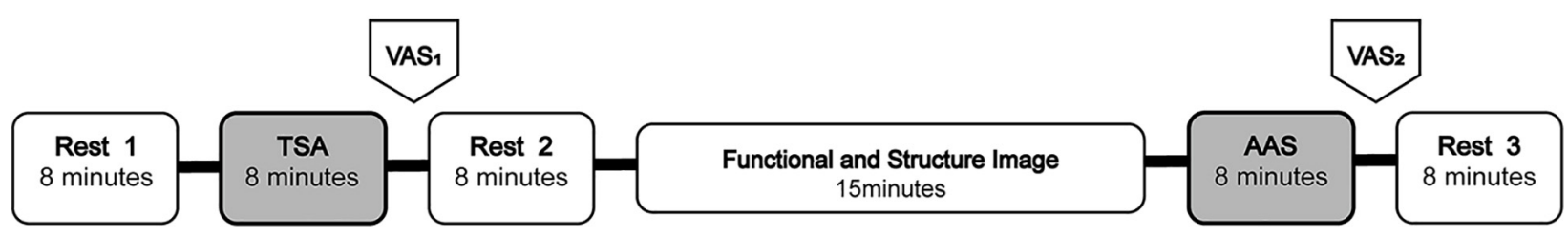

FIGURE 2 | fMRI scanning paradigm. The order of TSA and AAS runs was pseudo-randomized across subjects. After TAS or AAS stimulation run (needles withdrawal), rest runs were conducted. During rest runs, subjects were asked to lie quietly, keep their eyes closed and stay awake. The TSA and AAS portions of the scan session were separated by functional and structure image scan lasting 15 min.

\section{Pain Assessment}

A self-reported VAS was used to evaluate the current pain intensity experienced by each participant immediately after the TSA and AAS of the scan session. VAS rating was accomplished by the use of a custom-built MR-compatible handheld rotating knob, which was connected to a visual display projecting an intensity scale. The knob was set to traverse the entire rating range with minimal thumb finger twisting. The device was held in the right arm of participant. The VAS took $<1 \mathrm{~min}$ to complete. The scale was comprised of a $10 \mathrm{~cm}$ horizontal line, extending from " 0 " on the left indicating no pain, to " 10 " on the right indicating the most severe pain imaginable. Participants were instructed to mark a " $x$ " on the line according to the pain intensity they experienced currently. The length (in centimeters) from" " to the mark was recorded as their pain score.

\section{Functional Magnetic Resonance Imaging Data Analysis}

MRI data were processed in MATLAB (R2014), using SPM12 $^{2}$ and RESTplus ${ }^{3}$. The raw data were preprocessed as follows: the first 10 brain volumes were removed, slice timing and head motion correction were applied, functional and structural images were realigned, the structural image was segmented, and all data were spatially normalized into Montreal Neurological Institute (MNI) space, resampled at $3 \times 3 \times 3 \mathrm{~mm}$, and spatially smoothed using a Gaussian kernel of $6 \mathrm{~mm}$ full-width half-maximum (FWHM). Finally, the statistical model included regression of covariates (including Friston-24 head movement parameters, and white matter and cerebrospinal fluid time courses) and removal of the linear trend.

All BOLD signal fluctuations below the Nyquist frequency $(0.25 \mathrm{~Hz}$ in the present study) were delineated into six frequency bands: norm-1 $(0.01-0.1 \mathrm{~Hz})$, norm-2 $(0.01-0.08 \mathrm{~Hz})$, slow$5(0.01-0.027 \mathrm{~Hz})$, slow-4 $(0.027-0.073 \mathrm{~Hz})$, slow-3 (0.073$0.198 \mathrm{~Hz}$ ), and slow-2 $(0.198-0.25 \mathrm{~Hz})$ (Buzsáki and Draguhn, 2004). The whole-brain level of Amplitude of Low-Frequency Fluctuation (ALFF) was calculated within each frequency band. These ALFF values were converted to $\mathrm{z}$-score standardized values (zALFF) before statistical analysis.

${ }^{2}$ www.fil.ion.ucl.ac.uk/spm

${ }^{3}$ http://restfmri.net/forum/REST_V1.3

\section{Statistical Analyses}

Demographic data and VAS pain intensity scores were visualized using Graphpad prism $8^{4}$. Descriptive statistics were presented as mean \pm standard deviation. Here we used a linear mixedeffect modeling (LME) to examine the mean ALFF values of AAS and TSA between patients and healthy controls. LME is a flexible modeling approach that handles complex experimental designs and has been widely used in fMRI statistical analysis (Chen et al., 2013; Jo et al., 2021; Yang et al., 2021). The difference in VAS score between TSA and AAS conditions was determined using a twotailed paired $t$-test and significance considered at a threshold of 0.05 . The main effects of group (patients vs. healthy controls) and condition (AAS vs. TSA), and any interaction between the two were determined using a mixed-effects analysis in DPABI (V4.1) ${ }^{3}$ toolbox. Results were corrected based on Gaussian random field theory (GRF) $(z>2.3, p<0.01$, cluster $>20$ voxels). Post hoc analyses were performed when a significant interaction effect was detected. Associations between zALFF at the cluster peak coordinates and VAS scores were explored by calculating the Pearson correlation coefficient.

\section{RESULTS}

\section{Participants}

Fifty two participants (27 patients with CLBP and 25 volunteers) were recruited from Zhejiang Integrated Traditional and Western Medicine Hospital. 12 patients dropped out during the experimental period. Three participants could not tolerate the scanning session. Two participants were not imaged during acupuncture stimuli due to claustrophobia. Three participants terminated the study due to excessive fear. One subject was removed for suspected sub-clinical neuropathy. Three participants voluntarily withdrew. A final total of 40 patients (20 in the CLBP and 20 in the healthy subjects group) completed all the clinical assessments and imaging scans. In addition, one patient and one control were excluded because of severe head movement during fMRI. Therefore, data from 19 ( $n=12$ men) patients and 19 ( $n=11$ men) healthy subjects comprised the final dataset for analysis. All the participants reported no obvious needle sensation during acupuncture. The basic characteristics of

${ }^{4}$ www.graphpad.com/scientific-software/prism/ 
TABLE 1 | Characteristics of included participants.

\begin{tabular}{lcccc}
\hline & \multicolumn{2}{c}{ Group } & t/ $\chi^{2}$ & P-value \\
\cline { 2 - 4 } & Healthy control & Patient & & \\
\hline Gender (M/F) & $11 / 8$ & $12 / 7$ & 0.11 & 0.74 \\
Age (years) & $38.95 \pm 15.45$ & $46.61 \pm 7.35$ & 1.66 & 0.11 \\
Weight (kg) & $66.18 \pm 10.18$ & $64.05 \pm 5.63$ & 0.80 & 0.43 \\
Height (cm) & $168.10 \pm 7.33$ & $165.40 \pm 7.86$ & 1.11 & 0.27 \\
Pain duration & - & $9.00 \pm 7.72$ & - & - \\
\hline
\end{tabular}

patients with CLBP were as follows (Table 1): age $46 \pm 7$ years, height $165.4 \pm 7.9 \mathrm{~cm}$, weight $64.05 \pm 5.63 \mathrm{~kg}$. There were no significant differences in the above demographic measures between the two participant groups $(P>0.05)$, but duration of lower back pain in patients was $9.0 \pm 7.7$ years.

\section{Visual Analog Scale Pain Intensity Scores}

At a group level, the VAS scores of pain in patients were significantly $(t=6.10, P=0.001)$ lower after AAS $(3.50 \pm 2.23)$ in comparison to after TSA $(5.64 \pm 1.98)$. Duration of experiencing lower back pain was negatively correlated with the degree to which the VAS pain intensity score differed between the two conditions $(r=-0.52, P=0.02)$. All of healthy subjects were scored with VAS $\leq 1$ at the two observation points. There was no significant difference noticed in the VAS scores of pain after AAS in comparison to after TSA $(P>0.05)$.

\section{Multi-Frequency Band Z-Score Standardized Values}

As shown in Figure 3, at a group level, zALFF following AAS showed significant differences in several brain areas compared with that after TSA. These differences showed both similarities and differences when compared between frequency bands.

As shown in Table 2, zALFF within all frequency bands increased in the medial prefrontal cortex and decreased in the cerebellum, posterior cingulate cortex, and parahippocampal gyrus. For all frequency bands except the slow-5 band, zALFFs decreased in the right insular cortex. Except for the norm-2 and slow- 5 bands, zALFF increased in the right superior temporal gyrus. zALFF within norm-2, slow-3, and slow-2 bands decreased in the right precentral and postcentral gyrus, and amygdala. zALFF in the slow-2 band decreased in the left insula, amygdala, and precentral gyrus. Further, zALFF within each frequency band did not show any statistically significant differences with the main effects of participant group or any interaction between group and treatment.

The area over which each zALFF frequency band was present was determined. Following this, the area where all frequency bands overlapped was also determined. The peak coordinates of this area were found in the medial prefrontal cortex (mPFC; $x=-6, y=54, z=-21)$ and cerebellum/posterior cingulate cortex (PCC) $(x=0, y=-42, z=-15)$. The peak coordinate of the overlap between all frequency bands except the slow5 band was found to be in the right insula $(x=39, y=6$, $z=-21)$. Individual zALFF values for all frequency bands in

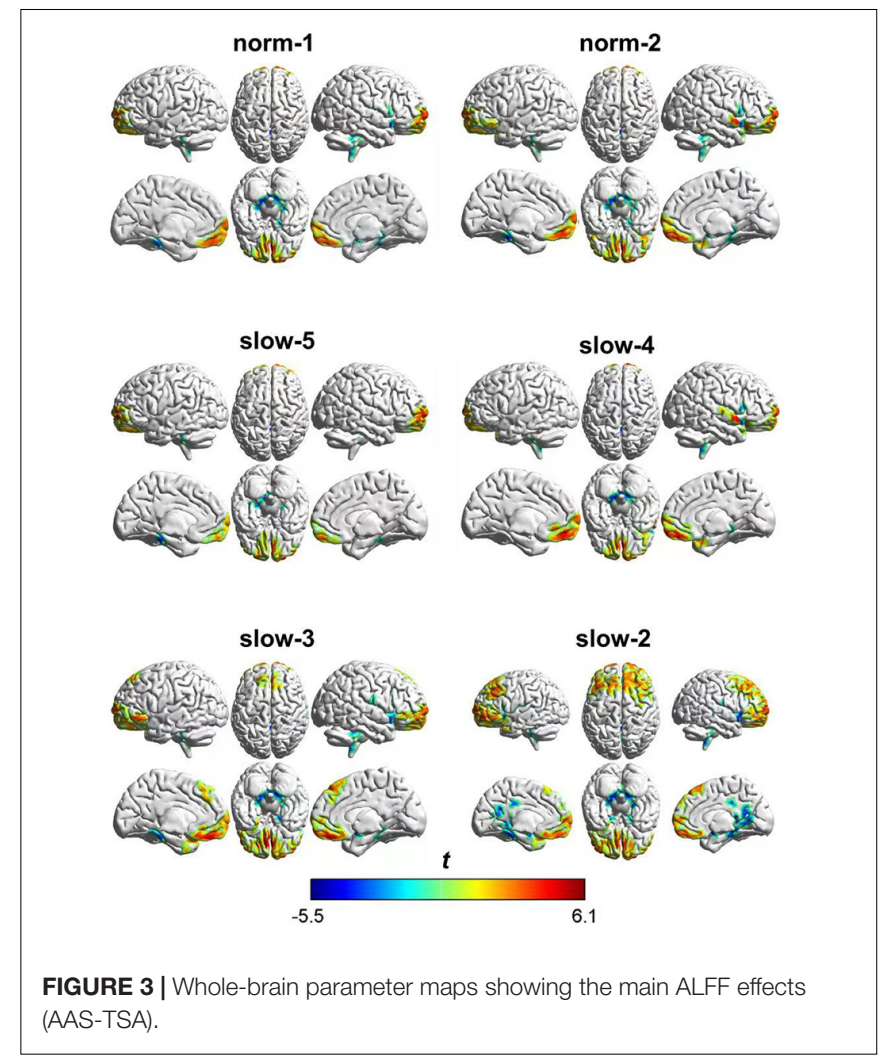

mPFC, cerebellum/PCC, and right and left insula $(x=-45, y=-$ $18, z=3$ ) are presented in Figure 4. We also re-analyzed these data using a voxel level of $p<0.001$ at the whole brain level. Clusters with smaller size located in the medial frontal cortex, cerebellum and the right insular cortex passed the correction threshold. The peak MNI coordinates of them were almost the same as that of clusters reported using threshold of a voxel level of $p<0.01$ (Supplementary Materials).

\section{The Relationship Between Pain and Z-Score Standardized Values}

As shown at the top of Figure 5, there was a significant association between the above zALFF effects and VAS pain intensity scores following AAS. Specifically, slow-5 frequency band zALFF in the cerebellum was significantly positively correlated with VAS score after AAS $(r=0.65, P=0.003)$, and negatively correlated with the change in VAS score between the two treatments ( $r=-0.53, P=0.02)$. Meanwhile, slow-3 frequency band zALFF in the cerebellum was also positively correlated with VAS score after AAS $(r=0.48, P=0.04)$. The zALFF within the norm-2 $(r=-0.48, P=0.04)$ and slow $-4(r=-0.48, P=0.04)$ frequency bands in the right insula showed negative correlations with changes in VAS score after AAS.

\section{DISCUSSION}

AAS is a form of acupuncture therapy that does not produce any needle sensation. Its stimulation is mild and mimics a tactile 
TABLE 2 | The ALFF results of main effects (AAS-TSA).

\begin{tabular}{|c|c|c|c|c|c|c|c|}
\hline \multirow[t]{2}{*}{ Band } & \multirow[t]{2}{*}{ Area } & \multirow[t]{2}{*}{ BA } & \multirow[t]{2}{*}{ Cluster size } & \multirow[t]{2}{*}{$t$-value } & \multicolumn{3}{|c|}{ MNI coordinate } \\
\hline & & & & & $\mathbf{x}$ & $\mathbf{y}$ & $\mathbf{Z}$ \\
\hline \multirow[t]{3}{*}{ Norm-1 } & mPFC & 10,11 & 1,222 & 4.99 & -6 & 54 & -21 \\
\hline & Cerebellum, PCC, parahippocampus & 27,30 & 655 & -5.48 & 3 & -39 & -15 \\
\hline & Right insula & 13 & 421 & -5.31 & 39 & -6 & 21 \\
\hline \multirow[t]{4}{*}{ Norm-2 } & $\mathrm{mPFC}$ & 10,11 & 1,199 & 5.21 & -6 & 54 & -21 \\
\hline & Right superior temporal gyrus & 38 & 231 & 4.88 & 60 & 9 & -6 \\
\hline & Cerebellum, PCC, parahippocampus & 27,30 & 572 & -5.71 & 3 & -39 & -15 \\
\hline & Right insula, precentral gyrus, postcentral gyrus, amygdala & $13,6,24$ & 346 & -5.35 & 39 & -6 & 21 \\
\hline \multirow[t]{2}{*}{ Slow-5 } & $\mathrm{mPFC}$ & 10,11 & 1,009 & 5.06 & -30 & 66 & 0 \\
\hline & Cerebellum, PCC, parahippocampus & 27,30 & 288 & -5.06 & 21 & -33 & -27 \\
\hline \multirow[t]{4}{*}{ Slow-4 } & mPFC & $10,11,34$ & 890 & 5.12 & -6 & 54 & -21 \\
\hline & Right superior temporal gyrus & 38,22 & 258 & 5.19 & 57 & 9 & -9 \\
\hline & Cerebellum, PCC, parahippocampus & 30,25 & 454 & -5.44 & 3 & -39 & -15 \\
\hline & Right insula & 13,6 & 253 & -4.84 & 39 & -6 & 21 \\
\hline \multirow[t]{4}{*}{ Slow-3 } & mPFC, Right superior temporal gyrus & $10,11,38$ & 1,622 & 6.10 & 0 & 39 & -24 \\
\hline & & 8,6 & 301 & 4.18 & 9 & 24 & 60 \\
\hline & Cerebellum, PCC, parahippocampus & 30,35 & 629 & -4.75 & 27 & -39 & -30 \\
\hline & Right insula, precentral gyrus, postcentral gyrus, amygdala & $13,4,722,3,834,6$ & 448 & -4.58 & 51 & 9 & 6 \\
\hline \multirow[t]{4}{*}{ Slow-2 } & mPFC, Right superior temporal gyrus & $6,8-1,138,46$ & 3,327 & 5.16 & 12 & 36 & -24 \\
\hline & Cerebellum, PCC, parahippocampus & $23,2,730$ & 1,358 & -5.43 & 15 & -36 & -15 \\
\hline & Right insula, precentral gyrus, postcentral gyrus, amygdala & $13,4,738,2,234,6$ & 764 & -5.12 & 45 & 0 & 3 \\
\hline & Left insula, precentral gyrus, amygdala & $13,2,234,4,144$ & 439 & -4.68 & -45 & -18 & 3 \\
\hline
\end{tabular}

BA, Brodmann; MNI, Montreal Neurological Institute; mPFC, medial prefrontal cortex; PCC, posterior cingulate cortex.

sensation on the skin. Therefore, AAS may be useful in the investigation of brain responses associated with acupuncture analgesia due to the ability to exclude or minimize the confound from forms of traditional acupuncture that are associated with stimulation from Deqi needle insertion. The current study used rs-fMRI to investigate whole-brain ALFF changes following AAS, and further explored the relationship between these changes and self-reported VAS pain intensity scores. Previous functional brain imaging studies investigating acupuncture have focused its effects either in healthy subjects or in patients with a specific diagnosis. To our knowledge, the present study was the first to collect and analyze functional brain imaging data following AAS in both patients and healthy subjects. The main results showed that in contrast to tactile sham acupuncture (TSA), self-reported pain intensity in patients with CLBP significantly decreased after AAS. Meanwhile, ALFF in sub-band frequencies ranging from 0.01 to $0.25 \mathrm{~Hz}$ was consistently modulated within nodes of the DMN (group-level increases in the mPFC, and decreases in the cerebellum/PCC/parahippocampus) in both patients and healthy subjects. Decreased ALFF in the bilateral insular cortex (nodes of the salience network) showed frequency-specific modulations. Furthermore, pain intensity experienced by patients showed a close relationship with the functional responses within specific bands in the right insula and cerebellum.

Acupuncture stimulation induces widespread responses within DMN (Zhang et al., 2019; Zou et al., 2019). Results from studies using a within-group design indicated that the BOLD oscillations in the $0.01-0.1 \mathrm{~Hz}$ frequency band in the mPFC and PCC reflect acupuncture stimulation at different acupoints (including Guangming, Kunlun, and Jiaoxin) near the lateral malleolus of healthy subjects (Liu et al., 2009; Qin et al., 2011; Deng et al., 2016; Sun et al., 2019). Zhu et al. (2015) conducted a TSA-controlled (placebo-controlled) study in healthy subjects, reporting that acupuncture administered at the Taixi acupoint resulted in modulating the fractional ALFF (fALFF) in the $0.01-0.1 \mathrm{~Hz}$ frequency band in the form of a decrease in the mPFC and cerebellum. Further, a study by Li et al. (2014) reported a significant correlation between the modulation of functional connectivity within the DMN and the analgesic effect of acupuncture in patients with CLBP. The current study not only found similar patterns in the differential modulations of resting-state brain function between AAS and sham acupuncture in DMN but also demonstrated the consistency of the response of DMN oscillations within each frequency band in the range from 0.01 to $0.25 \mathrm{~Hz}$ both in healthy subjects and patients with CLBP. It is noteworthy that, compared with previous studies using manual acupuncture and electroacupuncture, which both induce a Deqi sensation, AAS, provides the absolute minimum stimulus sensation, and is still sufficient to regulate DMN function in healthy subjects and patients with chronic pain. Therefore, as argued by Otti and Noll-Hussong (2012), DMN activity involves the transformation of a variety of basic physiological or pathological body states and provides a potential biomarker for use in the study of acupuncture analgesia mechanisms, including that of AAS. 


\section{MPFC}

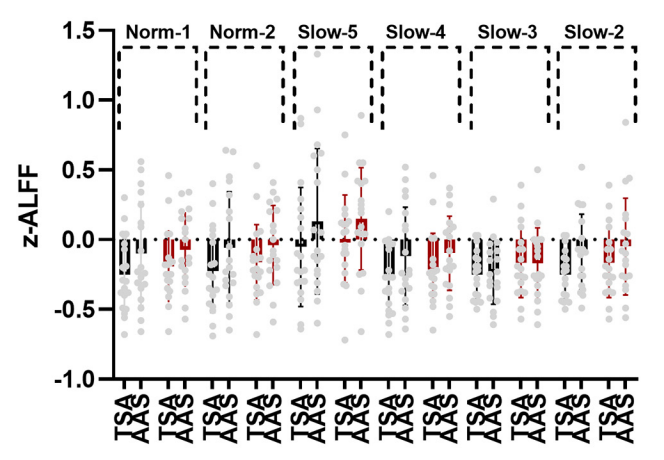

Right insula

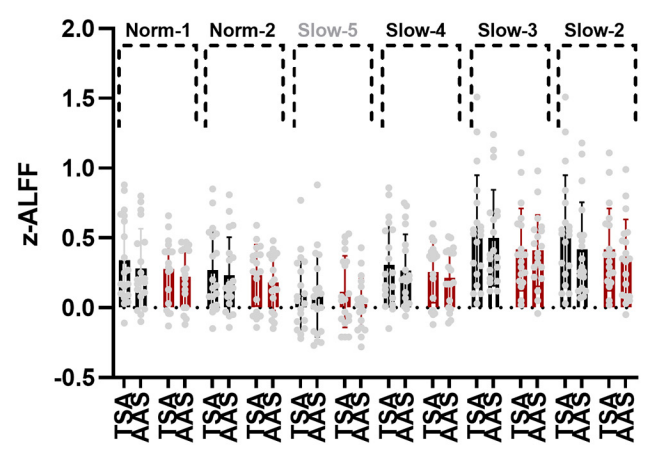

\section{Cerebellum/PCC}

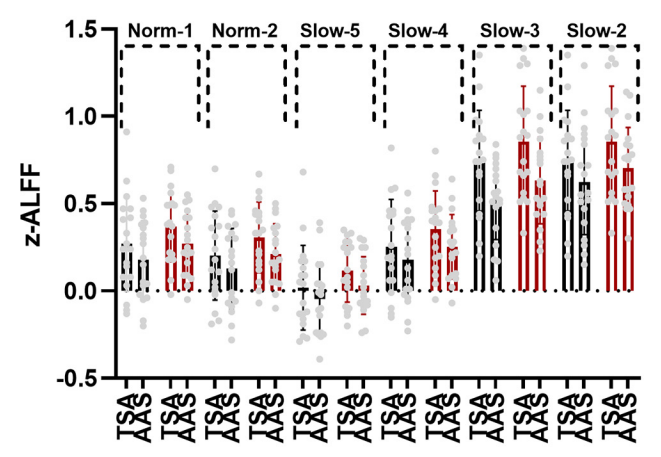

Left insula

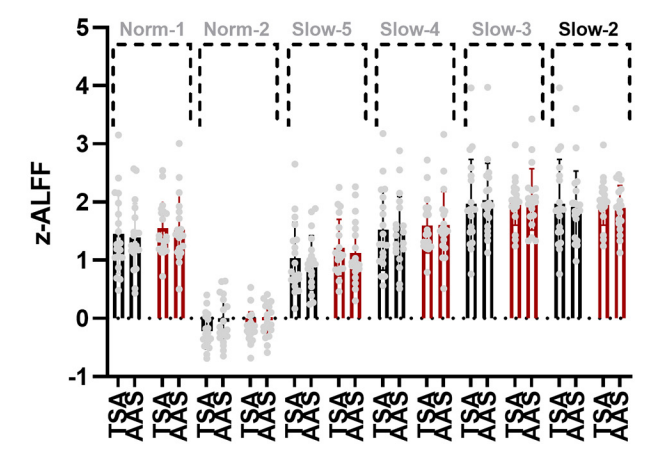

- Healthy controls

Patients

FIGURE 4 | Individual and group values of zALFF across frequency bands in mPFC, cerebellum/PCC, right and left insula (TSA, tactile sham acupuncture; AAS, ankle acupuncture stimulation, frequency bands in gray denote no difference in the present comparison).

Our study provides the characteristics of multi-band BOLD signal oscillation with ALFF as the index for the central response of AAS analgesia. In present study, frequency-specific resting-state activity correlated to AAS analgesia focused in the cerebellum and insular. It is shown that plasticity changes in the insular cortex have been frequently observed in patients with chronic back pain (Baliki et al., 2012; Kim et al., 2020). Studies using structural magnetic resonance imaging have reported reduced gray matter volumes in many forebrain regions including the insula. Studies using fMRI have reported elevations in the functional synchronization of BOLD signals in the 0.12$0.2 \mathrm{~Hz}$ range between the insula and $\mathrm{mPFC}$. A recent rs-fMRI study reported increased norm-2 band ALFF in the insular cortex associated with CLBP compared with that of healthy controls (Baliki et al., 2011; Fritz et al., 2016; Zhou et al., 2018). Results in the present and our previous research have found that decreases in ALFF across multiple frequency bands in the insula correlated with the analgesic effect of AAS (Xiang et al., 2019). This implies that the insular cortex may be an important target brain area related to the regulation of chronic pain by AAS (Xiang et al., 2019). Additionally, BOLD oscillations in the amygdala and somatosensory motor cortex have not yet been shown to correlate with the analgesic effects of AAS.
In present study, we identified frequency-specific resting-state activity in the cerebellum during the AAS stimulation. Notably, we found that norm-1 and norm-2 band ALFF patterns following acupuncture was very similar, but they did not completely overlapping, especially in the cerebellum. Only norm-2 band ALFF correlated to pain-relief effect of acupuncture. ALFF was originally defined based on norm $2(0.01-0.08 \mathrm{~Hz})$ band BOLD signal oscillation (Zang et al., 2007). However, norm 1 band oscillation was also analyzed in resting-state fMRI studies (Zuo et al., 2010). Both norm-1 and norm-2 bands are significant measures of resting-state brain activity and therefore should be considered in studies on acupuncture analgesia ( $\mathrm{Li}$ et al., 2017; Bao et al., 2018; Wu et al., 2018). To our knowledge, this was the first study to test the potential similarity and difference between norm 1 and norm 2 in acupuncture analgesia process. The cerebellum is generally considered to be a brain region involved in motor processing. Recent researches suggest that the cerebellum has also been implicated in non-motor, and even a number of integrative functions, including memory, associative learning, motor control (Schmahmann and Pandya, 1997; Ito, 2006). Notably, some fMRI studies show activation in the cerebellum during nociceptive processing (Borsook et al., 2008). Direct evidence from electrophysiological studies indicates 


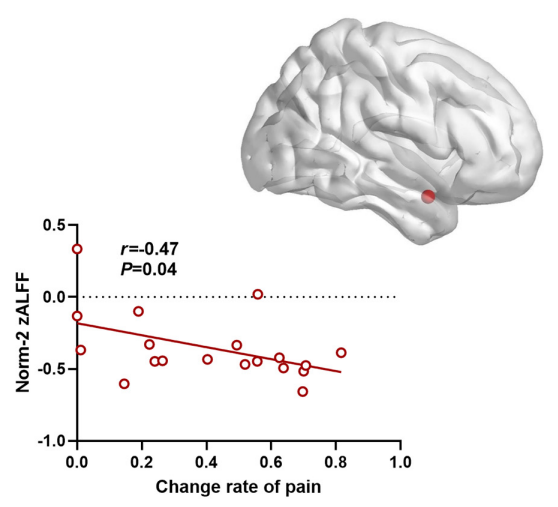

MNI: $x=39, y=6, z=-21$
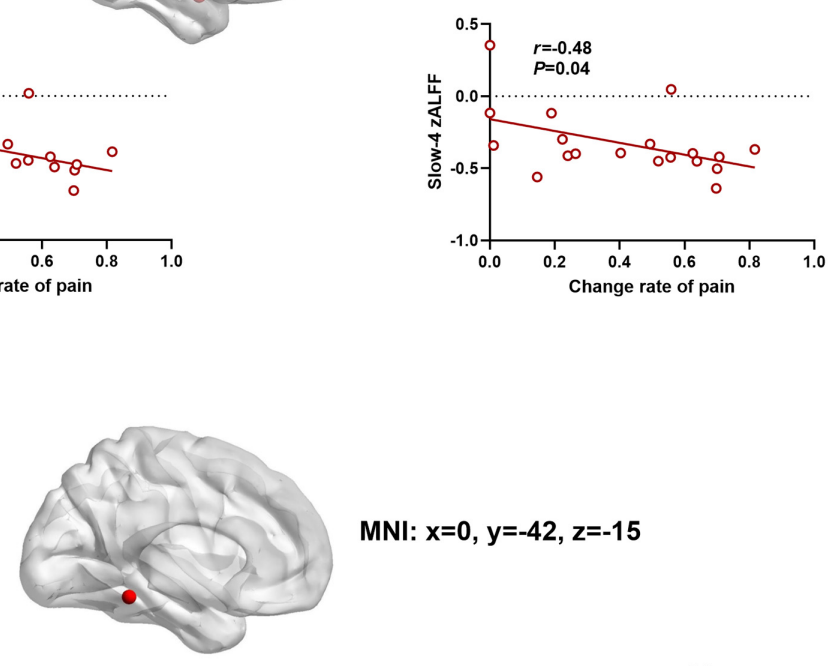

MNI: $x=0, y=-42, z=-15$
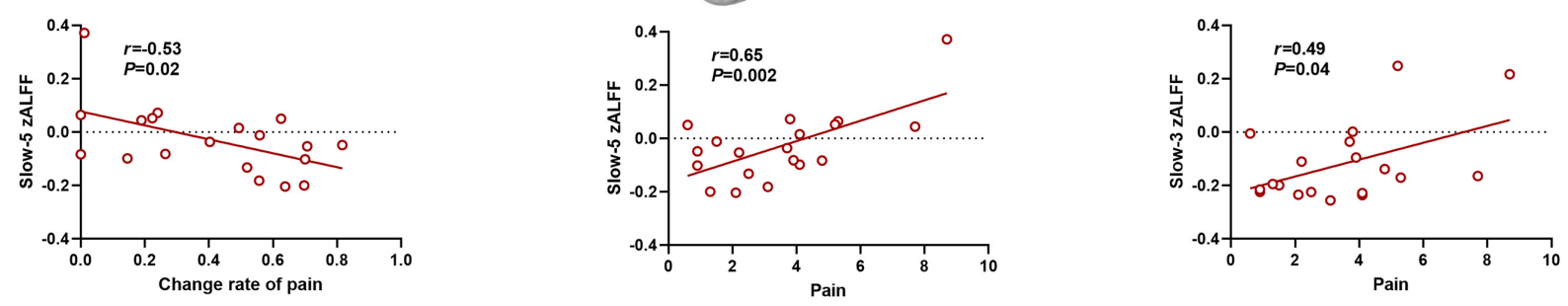

FIGURE 5 | The scatter plots between ZALFF and pain. Frequency-specific resting-state activity in the cerebellum and insular was correlated to AAS analgesia (the relationship were determined by Pearson coefficient value).

that the cerebellum receives nociceptive afferents (Ekerot et al., 1987; Jie and Pei-Xi, 1992). C-fiber nociceptors may act through mossy fibers to reach Purkinje cells in the cerebellum (Jie and Pei-Xi, 1992). Electrical stimulation of the intermediate portion of the anterior cerebellar lobe raised nociceptive thresholds to tail shock in monkeys (Siegel and Wepsic, 1974). Our results, consistent with other studies (Chae et al., 2013; Zheng et al., 2016; Bai et al., 2018), suggested that acupuncture stimulation modulated cerebellar activities during the process of pain relief. Current conceptualizations of pain in humans are multidimensional, mainly including the perception of the noxious stimulus, the affective features of pain, and cognitive components. Possible functional roles for the cerebellum relating to acupuncture modulation should be considered, including emotion, cognition, and motor control. Together with studies indicating a frequency-dependent modulation of BOLD signal oscillations in specific brain regions in patients with chronic pain (Wang et al., 2017; Rogachov et al., 2018; Gu et al., 2019), our study provides a practical rationale and mechanism for studying the frequency-dependence of BOLD oscillations in response to acupuncture analgesia.

In summary, the central mechanism underpinning the effects of AAS requires further explanation. This study has demonstrated a relationship between frequency-specific BOLD oscillations in the brain associated with ankle acupuncture analgesia and pain intensity scores in CLBP patients. The characteristics of BOLD signal oscillations across multiple frequency bands following AAS analgesia were demonstrated. Further work is necessary to further elucidate the mechanisms behind the effects of AAS through a combination of multiscale, multi-modal neuroimaging, animal models, and electrophysiological techniques.

\section{STUDY LIMITATIONS}

With regard to the study design and protocol, there are some limitations that need to be taken into account. Firstly, our results were observed in a small cohort of subjects. Therefore, further testing in clinical populations is warranted. Given that each different frequency band contributes uniquely to brain network integration in terms of physiological and pathological activities, the present studies of smaller size have yielded more information to help interpret localized BOLD changes associated with acupuncture analgesia and provided impetus to further investigations. These preliminary results may 
be of importance for the design of further confirmative studies. Secondly, washout period for AAS's effects remains unknown, although stimulus runs were separated by structural scanning for 15 min in present study.

\section{DATA AVAILABILITY STATEMENT}

The raw data supporting the conclusions of this article will be made available by the authors, without undue reservation.

\section{ETHICS STATEMENT}

The studies involving human participants were reviewed and approved by the Ethical Committee of the Center of Cognitive and Brain Disorders of Hangzhou Normal University. The patients/participants provided their written informed consent to participate in this study.

\section{REFERENCES}

Bai, L., Niu, X., Liu, Z., Chen, Z., Wang, X., Sun, C., et al. (2018). The role of insulacerebellum connection underlying aversive regulation with acupuncture. Mol. Pain 14:1744806918783457. doi: 10.1177/1744806918783457

Baliki, M. N., Baria, A. T., and Apkarian, A. V. (2011). The cortical rhythms of chronic back pain. J. Neurosci. 31, 13981-13990. doi: 10.1523/jneurosci.198411.2011

Baliki, M. N., Petre, B., Torbey, S., Herrmann, K. M., Huang, L., Schnitzer, T. J., et al. (2012). Corticostriatal functional connectivity predicts transition to chronic back pain. Nat. Neurosci. 15, 1117-1119. doi: 10.1038/nn.3153

Bao, C., Liu, P., Liu, H., Jin, X., Shi, Y., Wu, L., et al. (2018). Difference in regional neural fluctuations and functional connectivity in Crohn's disease: a restingstate functional MRI study. Brain Imaging Behav. 12, 1795-1803. doi: 10.1007/ s11682-018-9850-z

Berman, B. M., Langevin, H. M., Witt, C. M., and Dubner, R. (2010). Acupuncture for chronic low back pain. N. Engl. J. Med. 363, 454-461. doi: 10.1056/ NEJMct0806114

Borsook, D., Moulton, E. A., Tully, S., Schmahmann, J. D., and Becerra, L. (2008). Human cerebellar responses to brush and heat stimuli in healthy and neuropathic pain subjects. Cerebellum 7, 252-272. doi: 10.1007/s12311-0080011-6

Buzsáki, G., and Draguhn, A. (2004). Neuronal oscillations in cortical networks. Science 304, 1926-1929. doi: 10.1126/science.1099745

Chae, Y., Chang, D. S., Lee, S. H., Jung, W. M., Lee, I. S., Jackson, S., et al. (2013). Inserting needles into the body: a meta-analysis of brain activity associated with acupuncture needle stimulation. J. Pain 14, 215-222. doi: 10.1016/j.jpain.2012. 11.011

Chen, G., Saad, Z. S., Britton, J. C., Pine, D. S., and Cox, R. W. (2013). Linear mixedeffects modeling approach to FMRI group analysis. Neuroimage 73, 176-190. doi: 10.1016/j.neuroimage.2013.01.047

Deng, D., Duan, G., Liao, H., Liu, Y., Wang, G., Liu, H., et al. (2016). Changes in regional brain homogeneity induced by electro-acupuncture stimulation at the baihui acupoint in healthy subjects: a functional magnetic resonance imaging study. J. Altern. Complement. Med. 22, 794-799. doi: 10.1089/acm.2015.0286

Dhond, R. P., Yeh, C., Park, K., Kettner, N., and Napadow, V. (2008). Acupuncture modulates resting state connectivity in default and sensorimotor brain networks. Pain 136, 407-418. doi: 10.1016/j.pain.2008.01.011

Ekerot, C. F., Gustavsson, P., Oscarsson, O., and Schouenborg, J. (1987). Climbing fibres projecting to cat cerebellar anterior lobe activated by cutaneous A and $\mathrm{C}$ fibres. J. Physiol. 386, 529-538. doi: 10.1113/jphysiol.1987.sp016549

\section{AUTHOR CONTRIBUTIONS}

AX, XS, and SL conceived the study design. AX, MC, CQ, JR, and $\mathrm{CW}$ collected the data. AX analyzed the data. AX, MC, $\mathrm{CQ}$, XS, and SL helped to draft and revise the manuscript. All authors contributed to write the article and approved the submitted version.

\section{FUNDING}

This work was supported by National Natural Science Foundation of China (81873379).

\section{SUPPLEMENTARY MATERIAL}

The Supplementary Material for this article can be found online at: https://www.frontiersin.org/articles/10.3389/fnins. 2021.786490/full\#supplementary-material

Fritz, H. C., McAuley, J. H., Wittfeld, K., Hegenscheid, K., Schmidt, C. O., Langner, S., et al. (2016). Chronic back pain is associated with decreased prefrontal and anterior insular gray matter: results from a population-based cohort study. J. Pain 17, 111-118. doi: 10.1016/j.jpain.2015.10.003

Furlan, A. D., van Tulder, M. W., Cherkin, D. C., Tsukayama, H., Lao, L., Koes, B. W., et al. (2005). Acupuncture and dry-needling for low back pain. Cochrane Database Syst. Rev. Cd001351. doi: 10.1002/14651858.CD001351.pub2

Gao, Q., Xu, F., Jiang, C., Chen, Z., Chen, H., Liao, H., et al. (2016). Decreased functional connectivity density in pain-related brain regions of female migraine patients without aura. Brain Res. 1632, 73-81. doi: 10.1016/j.brainres.2015.12. 007

Gu, L., Hong, S., Jiang, J., Liu, J., Cao, X., Huang, Q., et al. (2019). Bidirectional alterations in ALFF across slow-5 and slow-4 frequencies in the brains of postherpetic neuralgia patients. J. Pain Res. 12, 39-47. doi: 10.2147/jpr.S179077

Harrisson, S. A., Stynes, S., Dunn, K. M., Foster, N. E., and Konstantinou, K. (2017). Neuropathic pain in low back-related leg pain patients: what is the evidence of prevalence, characteristics, and prognosis in primary care? A systematic review of the literature. J. Pain 18, 1295-1312. doi: 10.1016/j.jpain.2017.04.012

He, Z., Cui, Q., Zheng, J., Duan, X., Pang, Y., Gao, Q., et al. (2016). Frequencyspecific alterations in functional connectivity in treatment-resistant and sensitive major depressive disorder. J. Psychiatry Res. 82, 30-39. doi: 10.1016/ j.jpsychires.2016.07.011

Hipp, J. F., and Siegel, M. (2015). BOLD fMRI correlation reflects frequencyspecific neuronal correlation. Curr. Biol. 25, 1368-1374. doi: 10.1016/j.cub. 2015.03.049

Hoy, D., Brooks, P., Blyth, F., and Buchbinder, R. (2010). The epidemiology of low back pain. Best Pract. Res. Clin. Rheumatol. 24, 769-781. doi: 10.1016/j.berh. 2010.10.002

Hui, K. K., Liu, J., Makris, N., Gollub, R. L., Chen, A. J., Moore, C. I., et al. (2000). Acupuncture modulates the limbic system and subcortical gray structures of the human brain: evidence from fMRI studies in normal subjects. Hum. Brain Mapp. 9, 13-25. doi: 10.1002/(sici)1097-019320009:1<13::aid-hbm2<3.0.co;2-f

Ito, M. (2006). Cerebellar circuitry as a neuronal machine. Prog. Neurobiol. 78, 272-303. doi: 10.1016/j.pneurobio.2006.02.006

Jie, W., and Pei-Xi, C. (1992). Discharge response of cerebellar Purkinje cells to stimulation of C-fiber in cat saphenous nerve. Brain Res. 581, 269-272. doi: 10.1016/0006-8993(92)90717-n

Jo, S., Kim, H. C., Lustig, N., Chen, G., and Lee, J. H. (2021). Mixed-effects multilevel analysis followed by canonical correlation analysis is an effective fMRI tool for the investigation of idiosyncrasies. Hum. Brain Mapp. 42, 53745396. doi: 10.1002/hbm.25627 
Kim, H., Mawla, I., Lee, J., Gerber, J., Walker, K., Kim, J., et al. (2020). Reduced tactile acuity in chronic low back pain is linked with structural neuroplasticity in primary somatosensory cortex and is modulated by acupuncture therapy. Neuroimage 217:116899. doi: 10.1016/j.neuroimage.2020.116899

Konno, S. I., and Sekiguchi, M. (2018). Association between brain and low back pain. J. Orthop. Sci. 23, 3-7. doi: 10.1016/j.jos.2017.11.007

Li, J., Zhang, J. H., Yi, T., Tang, W. J., Wang, S. W., and Dong, J. C. (2014). Acupuncture treatment of chronic low back pain reverses an abnormal brain default mode network in correlation with clinical pain relief. Acupunct. Med. 32, 102-108. doi: 10.1136/acupmed-2013-010423

Li, Z., Zeng, F., Yin, T., Lan, L., Makris, N., Jorgenson, K., et al. (2017). Acupuncture modulates the abnormal brainstem activity in migraine without aura patients. Neuroimage Clin. 15, 367-375. doi: 10.1016/j.nicl.2017.05.013

Liu, P., Zhang, Y., Zhou, G., Yuan, K., Qin, W., Zhuo, L., et al. (2009). Partial correlation investigation on the default mode network involved in acupuncture: an fMRI study. Neurosci. Lett. 462, 183-187. doi: 10.1016/j.neulet.2009.07.015

Lou, Y. T., Li, X. L., Wang, Y., Ji, G. J., Zang, Y. F., Wang, J., et al. (2020). Frequency-specific regional homogeneity alterations in tourette syndrome. Front. Psychiatry 11:543049. doi: 10.3389/fpsyt.2020.543049

Marra, C., Pozzi, I., Ceppi, L., Sicuri, M., Veneziano, F., and Regalia, A. L. (2011). Wrist-ankle acupuncture as perineal pain relief after mediolateral episiotomy: a pilot study. J. Altern. Complement. Med. 17, 239-241. doi: 10.1089/acm.2010. 0256

Napadow, V., Makris, N., Liu, J., Kettner, N. W., Kwong, K. K., and Hui, K. K. (2005). Effects of electroacupuncture versus manual acupuncture on the human brain as measured by fMRI. Hum. Brain Mapp. 24, 193-205. doi: 10.1002/hbm. 20081

Otti, A., and Noll-Hussong, M. (2012). Acupuncture-induced pain relief and the human brain's default mode network-an extended view of central effects of acupuncture analgesia. Forsch. Komplementmed. 19, 197-201. doi: 10.1159/ 000341928

Qin, W., Bai, L., Dai, J., Liu, P., Dong, M., Liu, J., et al. (2011). The temporal-spatial encoding of acupuncture effects in the brain. Mol. Pain 7:19. doi: 10.1186/17448069-7-19

Rogachov, A., Cheng, J. C., Hemington, K. S., Bosma, R. L., Kim, J. A., Osborne, N. R., et al. (2018). Abnormal low-frequency oscillations reflect trait-like pain ratings in chronic pain patients revealed through a machine learning approach. J. Neurosci. 38, 7293-7302. doi: 10.1523/jneurosci.0578-18.2018

Schmahmann, J. D., and Pandya, D. N. (1997). The cerebrocerebellar system. Int. Rev. Neurobiol. 41, 31-60. doi: 10.1016/s0074-7742(08)60346-3

Siegel, P., and Wepsic, J. G. (1974). Alteration of nociception by stimulation of cerebellar structures in the monkey. Physiol. Behav. 13, 189-194. doi: 10.1016/ 0031-9384(74)90033-X

Sun, L., Chen, Y. Y., Fang, J. L., Hong, Y., Wang, Y., Xu, K., et al. (2019). Correlation between blood oxygen level dependent fMRI signal and GABA content in anterior cingulate cortex after acupuncture of Hegu (LI4). Zhen Ci Yan Jiu 44, 878-883. doi: 10.13702/j.1000-0607.190597

Urits, I., Burshtein, A., Sharma, M., Testa, L., Gold, P. A., Orhurhu, V., et al. (2019). Low back pain, a comprehensive review: pathophysiology, diagnosis, and treatment. Curr. Pain Headache Rep. 23:23. doi: 10.1007/s11916-019-0 $757-1$

Wang, X., Chan, S. T., Fang, J., Nixon, E. E., Liu, J., Kwong, K. K., et al. (2013). Neural encoding of acupuncture needling sensations: evidence from a FMRI study. Evid. Based Complement. Alternat. Med. 2013:483105. doi: 10.1155/2013/ 483105

Wang, Y., Xu, C., Zhai, L., Lu, X., Wu, X., Yi, Y., et al. (2017). Spatial-temporal signature of resting-state BOLD signals in classic trigeminal neuralgia. J. Pain Res. 10, 2741-2750. doi: 10.2147/jpr.S143734

Wang, Y., Xu, J., Zhang, Q., Zhang, Q., Yang, Y., Wei, W., et al. (2021). Immediate analgesic effect of acupuncture in patients with primary dysmenorrhea: a fMRI study. Front. Neurosci. 15:647667. doi: 10.3389/fnins.2021.647667

Wu, J. J., Lu, Y. C., Hua, X. Y., Ma, S. J., Shan, C. L., and Xu, J. G. (2018). Cortical remodeling after electroacupuncture therapy in peripheral nerve repairing model. Brain Res. 1690, 61-73. doi: 10.1016/j.brainres.2018.04.009

Xiang, A., Yu, Y., Jia, X., Ma, H., Liu, H., Zhang, Y., et al. (2019). The low-frequency BOLD signal oscillation response in the insular associated to immediate analgesia of ankle acupuncture in patients with chronic low back pain. J. Pain Res. 12, 841-850. doi: 10.2147/jpr.S189390
Yan, Y., Qian, T., Xu, X., Han, H., Ling, Z., Zhou, W., et al. (2020). Human cortical networking by probabilistic and frequency-specific coupling. Neuroimage 207:116363. doi: 10.1016/j.neuroimage.2019.116363

Yang, H., Chen, X., Chen, Z. B., Li, L., Li, X. Y., Castellanos, F. X., et al. (2021). Disrupted intrinsic functional brain topology in patients with major depressive disorder. Mol. Psychiatry doi: 10.1038/s41380-021-01247-2

Zang, Y. F., He, Y., Zhu, C. Z., Cao, Q. J., Sui, M. Q., Liang, M., et al. (2007). Altered baseline brain activity in children with ADHD revealed by restingstate functional MRI. Brain Dev. 29, 83-91. doi: 10.1016/j.braindev.2006.0 7.002

Zeng, K., Dong, H. J., Chen, H. Y., Chen, Z., Li, B., and Zhou, Q. H. (2014). Wristankle acupuncture for pain after transcatheter arterial chemoembolization in patients with liver cancer: a randomized controlled trial. Am. J. Chin. Med. 42, 289-302. doi: 10.1142/s0192415x14500190

Zhang, R., Lao, L., Ren, K., and Berman, B. M. (2014). Mechanisms of acupunctureelectroacupuncture on persistent pain. Anesthesiology 120, 482-503. doi: 10. 1097/aln.0000000000000101

Zhang, X. (1997). Wrist-Ankle Acupuncture. Beijing: People's Military Medical Press.

Zhang, Y., Wang, Z., Du, J., Liu, J., Xu, T., Wang, X., et al. (2021). Regulatory effects of acupuncture on emotional disorders in patients with menstrual migraine without aura: a resting-state fMRI study. Front. Neurosci. 15:726505. doi: 10. 3389/fnins.2021.726505

Zhang, Y., Zhang, H., Nierhaus, T., Pach, D., Witt, C. M., and Yi, M. (2019). Default mode network as a neural substrate of acupuncture: evidence, challenges and strategy. Front. Neurosci. 13:100. doi: 10.3389/fnins.2019.00100

Zheng, Y., Zhang, J., Wang, Y., Wang, Y., Lan, Y., Qu, S., et al. (2016). Acupuncture decreases blood pressure related to hypothalamus functional connectivity with frontal lobe, cerebellum, and insula: a study of instantaneous and short-term acupuncture treatment in essential hypertension. Evid. Based Complement. Alternat. Med. 2016:6908710. doi: 10.1155/2016/6908710

Zhou, F., Gu, L., Hong, S., Liu, J., Jiang, J., Huang, M., et al. (2018). Altered low-frequency oscillation amplitude of resting state-fMRI in patients with discogenic low-back and leg pain. J. Pain Res. 11, 165-176. doi: 10.2147/jpr. S151562

Zhu, B., Wang, Y., Zhang, G., Ouyang, H., Zhang, J., Zheng, Y., et al. (2015). Acupuncture at KI3 in healthy volunteers induces specific cortical functional activity: an fMRI study. BMC Complement. Altern. Med. 15:361. doi: 10.1186/ s12906-015-0881-3

Zhu, L. B., Chan, W. C., Lo, K. C., Yum, T. P., and Li, L. (2014). Wrist-ankle acupuncture for the treatment of pain symptoms: a systematic review and metaanalysis. Evid. Based Complement. Alternat. Med. 2014:261709. doi: 10.1155/ 2014/261709

Zou, Y., Tang, W., Li, X., Xu, M., and Li, J. (2019). Acupuncture reversible effects on altered default mode network of chronic migraine accompanied with clinical symptom relief. Neural. Plast. 2019:5047463. doi: 10.1155/2019/504 7463

Zuo, X. N., Di Martino, A., Kelly, C., Shehzad, Z. E., Gee, D. G., Klein, D. F., et al. (2010). The oscillating brain: complex and reliable. Neuroimage 49, 1432-1445. doi: 10.1016/j.neuroimage.2009.09.037

Conflict of Interest: The authors declare that the research was conducted in the absence of any commercial or financial relationships that could be construed as a potential conflict of interest.

Publisher's Note: All claims expressed in this article are solely those of the authors and do not necessarily represent those of their affiliated organizations, or those of the publisher, the editors and the reviewers. Any product that may be evaluated in this article, or claim that may be made by its manufacturer, is not guaranteed or endorsed by the publisher.

Copyright (C) 2021 Xiang, Chen, Qin, Rong, Wang, Shen and Liu. This is an openaccess article distributed under the terms of the Creative Commons Attribution License (CC BY). The use, distribution or reproduction in other forums is permitted, provided the original author(s) and the copyright owner(s) are credited and that the original publication in this journal is cited, in accordance with accepted academic practice. No use, distribution or reproduction is permitted which does not comply with these terms. 